\title{
Establishment and characterization of two 5-fluorouracil- resistant hepatocellular carcinoma cell lines
}

\author{
KAZUYA UCHIBORI ${ }^{1,2}$, ATSUSHI KASAMATSU ${ }^{3,4}$, MASAHIKO SUNAGA $^{5}$, SATOSHI YOKOTA ${ }^{3}$, \\ TOMOYA SAKURADA ${ }^{1}$, ERIKO KOBAYASHI ${ }^{1}$, MASAHARU YOSHIKAWA ${ }^{2}$, KATSUHIRO UZAWA ${ }^{3,4}$, \\ SHIRO UEDA $^{2}$, HIDEKI TANZAWA ${ }^{3,4}$ and NOBUNORI SATO ${ }^{1}$ \\ ${ }^{1}$ Department of Clinical Education and Research, Graduate School of Pharmaceutical Sciences, Chiba University; \\ Departments of ${ }^{2}$ Medicine and Clinical Oncology and ${ }^{3}$ Clinical Molecular Biology, Graduate School of Medicine, \\ Chiba University; ${ }^{4}$ Division of Oral-Maxillofacial Surgery, Chiba University Hospital, 1-8-1 Inohana, Chuo-ku, \\ Chiba 260-8670; ${ }^{5}$ Chiba Central Medical Center, 1835-1 Kasori-cho, Wakaba-ku, Chiba 264-0017, Japan
}

Received September 28, 2011; Accepted November 22, 2011

DOI: 10.3892/ijo.2011.1300

\begin{abstract}
Fluorouracil (5-FU) chemotherapy is the first choice treatment for advanced hepatocellular carcinoma (HCC), and resistance is the major obstacle to successful treatment. Recent studies have reported that epithelial-to-mesenchymal transition (EMT) is associated with chemoresistance in cancers. We speculated that EMT and 5-FU metabolism are related to the mechanism of 5-FU resistance. First, two 5-FU-resistant cell lines, HLF-R4 and HLF-R10, were established from the HLF undifferentiated human HCC cell line. Whereas cell growth was similar in the HLF and HLF-R cell lines, HLF-Rs are about 4and 10-fold more resistant compared with the HLF cells; thus, we named these cell lines HLF-R4 and HLF-R10, respectively. The terminal deoxyribonucleotidyl transferase-mediated dUTP nick end labeling assay also showed a dramatically decreased number of apoptotic cells in the HLF-Rs after treatment with 5-FU. We next assessed the characteristics of the HLF, HLF-R4 and HLF-R10 cells. Consistent with our hypothesis, the HLF-Rs had typical morphologic phenotypes of EMT, loss of cell-cell adhesion, spindle-shaped morphology and increased formation of pseudopodia. Real-time quantitative reverse transcriptase polymerase chain reaction data showed downregulated E-cadherin and upregulated Twist-1 and also indicated that EMT changes occurred in the HLF-Rs. We also found decreased ribonucleotide reductase and increased multidrug resistance protein 5 genes in the HLF-R cells. Our results suggested that the metabolism of EMT and 5-FU has important roles in 5-FU chemoresistance in the HLF-R cells, and that the
\end{abstract}

Correspondence to: Dr Atsushi Kasamatsu, Department of Molecular Biology, Graduate School of Medicine, Chiba University, 1-8-1 Inohana, Chuo-ku, Chiba 260-8670, Japan

E-mail:kasamatsua@faculty.chiba-u.jp

Key words: 5-fluorouracil, drug resistance, hepatocellular carcinoma, epithelial-to-mesenchymal transition
HLF-R cells would be useful in vitro models for understanding the 5-FU-resistant mechanisms in HCC.

\section{Introduction}

Hepatocellular carcinoma (HCC) is one of the most frequently occurring malignant tumors and a common cause of cancer mortality worldwide (1). The results of surgical treatments for advanced HCC, such as liver resection or liver transplantation, have not been satisfactory. To date, chemotherapy, including transcatheter arterial infusion, is the first choice for advanced HCC (2,3). 5-Fluorouracil (5-FU) is a commonly used chemotherapeutic agent that is effective for treating a wide variety of malignant tumors. However, the effectiveness of the chemotherapy is limited because of acquired or intrinsic drug resistance $(4,5)$.

The development of resistance to 5-FU appears to be a major impediment to the successful chemotherapy of human cancers. 5-FU decreases the biosynthesis of pyrimidine nucleotides by inhibiting thymidylate synthase, which catalyzes the ratelimiting step in DNA synthesis (6-10). Although the mechanism of resistance to 5-FU remains unclear, a possible mechanism is that alterations of plasma membrane proteins reduce the accumulation of 5-FU within tumor cells (11). Two studies also reported that epithelial-to-mesenchymal transition (EMT) was closely related to chemoresistance in the colorectal and pancreatic cancers $(12,13)$.

EMT is initially observed in embryonic development in which cells lose epithelial characteristics and gain mesenchymal properties to increase motility and invasiveness $(12,14)$. Previous research has suggested that EMT is also important in tumor progression, metastasis, and chemoresistance $(14,15)$ and is induced by growth factors, such as hepatocyte growth factor, transforming growth factor $\beta$, and epidermal growth factor, implicated in these processes (16).

Tissue culture systems have been established to study the biochemical, physiologic, and genetic bases of alterations that result in development of multidrug resistance. To understand drug resistance well, establishing cultured cell lines resistant to 
anticancer drugs is necessary. In the current study, we established two 5-FU-resistant cell lines, HLF-R4 and HLF-R10, from an HCC cell line and investigated for the first time the mechanisms of 5-FU resistance including EMT.

\section{Materials and methods}

Cell lines. The HLF cell line, derived from undifferentiated human hepatocellular carcinoma, was obtained from the Japanese Cancer Research Resources Bank and maintained in Dulbecco's modified Eagle's medium (DMEM) (Gibco, Carlsbad, CA) supplemented with $10 \%$ fetal bovine serum (FBS) (Gibco) and 100 units/ml penicillin and streptomycin (Gibco). According to previously described methods (17-20), two 5-FU-resistant sublines, HLF-Rs, were established by repeated subcultures in the presence of stepwise-increases in concentrations of 5-FU (5, 7.5, 10 and $20 \mu \mathrm{M}$ ) (Wako, Osaka, Japan). Because the chemoresistance of both cell lines are 4- and 10-fold increased, respectively, the sublines were named HLF-R4 and HLF-R10, and they are fully resistant to 5-FU and can grow exponentially in the presence of 10 and $20 \mu \mathrm{M}$ of 5-FU. The HLF-Rs showed no loss of resistance even after 2 months of culture in a drug-free medium.

Cell growth. To investigate cell growth in the HLF, HLF-R4, and HLF-R10 cells, we performed a cell proliferation assay. The experiments were carried out for $168 \mathrm{~h}$, and the number of cells were counted every $24 \mathrm{~h}$. The cells in three samples were trypsinized and counted using a hemocytometer at the indicated time points.

Chemosensitivity assay. The cells were seeded at a concentration of $2.0 \times 10^{3}$ in each well of a $96-$ well plate in DMEM containing $10 \%$ FBS. After $24 \mathrm{~h}$, culture medium was replaced with DMEM with $10 \%$ FBS and various concentrations of 5-FU $(6.25,12.5,25,50,100,200,400,800$ and $1600 \mu \mathrm{M})$. After further incubation for $72 \mathrm{~h}$, a cell viability assay was carried out using the Cell Counting kit-8 according to the manufacturer's instructions (Dojindo, Kumamoto, Japan). Six wells were used for each drug concentration and the experiment was replicated three times. The $50 \%$ inhibitory concentration $\left(\mathrm{IC}_{50}\right)$ was calculated from the survival curves.

TUNEL assay. The cells were seeded at a concentration of $5.0 \times 10^{5}$ on Lab-TekII chamber slides (Nalge Nunc International, Rochester, NY) in DMEM containing 10\% FBS. After incubation for $24 \mathrm{~h}$, the culture medium was replaced with DMEM with $10 \% \mathrm{FBS}$ and $70 \mu \mathrm{M}$ of 5-FU. After further incubation for $24 \mathrm{~h}$, the cells on the chamber slides were washed twice with phosphate buffered saline, air dried, and fixed in $4 \%$ paraformaldehyde at room temperature for $30 \mathrm{~min}$. The terminal deoxyribonucleotidyl transferase-mediated dUTP nick end labeling (TUNEL) assay was performed using an In Situ Apoptosis Detection kit according to the manufacturer's instructions (Takara, Tokyo, Japan). The cells were viewed and photographed under a fluorescence microscope.

Preparation of cDNA. Total RNA was isolated using an RNeasy Mini kit (Qiagen, Chatsworth, CA) according to the manufacturer's instructions. cDNA was generated from $5 \mu \mathrm{g}$ of total
RNA using an Ominiscript RT kit (Qiagen) and random hexamer (Sigma Genosys, Ishikari, Japan).

$m R N A$ expression analysis. Real-time quantitative reverse transcriptase polymerase chain reaction (qRT-PCR) was performed to evaluate the expression levels of five target genes in HLF and its derivatives. qRT-PCR was carried out with one method using a LightCycler FastStart DNA Master SYBR Green 1 kit (Roche, Mannheim, Germany). The specific primers for the target genes are listed in Table I. Amplified products were analyzed by $3 \%$ agarose gel electrophoresis to ascertain size and purity. The PCR reactions using the LightCycler apparatus were performed in a final volume of $20 \mu \mathrm{l}$ of a reaction mixture comprised of $2 \mu \mathrm{l}$ of FirstStart DNA Master SYBR Green I mix, $3 \mathrm{mM} \mathrm{MgCl}$, and $1 \mu \mathrm{M}$ of the primers, according to the manufacturer's instructions. The reaction mixture was loaded into glass capillary tubes and subjected to an initial denaturation at $95^{\circ} \mathrm{C}$ for $10 \mathrm{~min}$, followed by 45 rounds of amplification at $95^{\circ} \mathrm{C}(10 \mathrm{sec})$ for denaturation, $62^{\circ} \mathrm{C}(10 \mathrm{sec})$ for annealing, and $72^{\circ} \mathrm{C}(10 \mathrm{sec})$ for extension, with a temperature slope of $20^{\circ} \mathrm{C} / \mathrm{sec}$. The transcript amounts for the target genes were estimated from the respective standard curves and normalized to the glyceraldehyde-3-phosphate dehydrogenase (GAPDH) transcript amount determined in corresponding samples.

Statistical analysis. The statistical significance was evaluated using the Mann-Whitney U test. $\mathrm{P}<0.05$ was considered statistically significant.

\section{Results}

Cell growth. To obtain 5-FU-resistant cells, the HLF cells were treated with increasing concentrations of 5-FU up to $20 \mu \mathrm{M}$, and two clones, designated HLF-R4 and HLF-R10, were established almost 1.5 years later. There were no significant differences in cellular proliferation among the HLF, HLF-R4, and HLF-R10 cells (Fig. 1).

Chemosensitivity assay. The $\mathrm{IC}_{50}$ data indicated that the 5-FU-resistance levels of the HLF-R4 cells $\left(\mathrm{IC}_{50}, 69.80 \mu \mathrm{M}\right)$ and HLF-R10 cells $\left(\mathrm{IC}_{50}, 193.47 \mu \mathrm{M}\right)$ were 3.9 - and 10.8-fold greater than that of the HLF cells ( $\left.\mathrm{IC}_{50}, 17.92 \mu \mathrm{M}\right)$ (Fig. 2).

TUNEL assay. TUNEL staining showed a dramatic increase in the number of cells that were stained green, which indicated that apoptosis occurred in HLF cells exposed to 5-FU, whereas 5-FU-induced apoptosis was prevented in the HLF-R4 and HLF-R10 cells (Fig. 3).

Cell morphology. The 5-FU-resistant cells, HLF-R4 and HLF-R10, were morphologically distinct from their parental cell line (Fig. 4). The resistant cells had loss of cell-cell adhesion, spindle-shaped morphology, and increased formation of pseudopodia. These changes are typical phenotypes of EMT.

Evaluation of the expression of genes putatively related to 5-FU resistance and EMT. Consistent with our hypothesis that EMT occurs in 5-FU-resistant cells, qRT-PCR showed decreased $E$-cadherin and increased Twist- 1 by in the HLF-Rs. Among the genes associated with 5-FU metabolism, we also 
Table I. Specific primers.

Forward $\left(5^{\prime}-3^{\prime}\right)$

Reverse (5'-3')

\begin{tabular}{lll}
\hline$R N R-R 1$ & AGAGAAGGAGAGGAACACAGCAG & AGCAAAGCCTTACCACCTCAAG \\
$R N R-$ R 2 & GCCCCTGTTAAGTGGTGAAATC & GCCAGAATAAGACACTGGGTGAC \\
MRP5 & TGAGACAGAAGCTCGATTCACC & AGGGAGGTTTTCTCGGTACCTC \\
E-cadherin & CTCTTCCAGGAACCTCTGTGATG & CCACACTGATGACTCCTGTGTTC \\
Twist 1 & GCCGGAGACCTAGATGTCATTG & CTATCAGAATGCAGAGGTGTGAGG \\
GAPDH & GAGCCAAAAGGGTCATCATCTC & GGTCATGAGTCCTTCCACGATAC \\
\hline
\end{tabular}

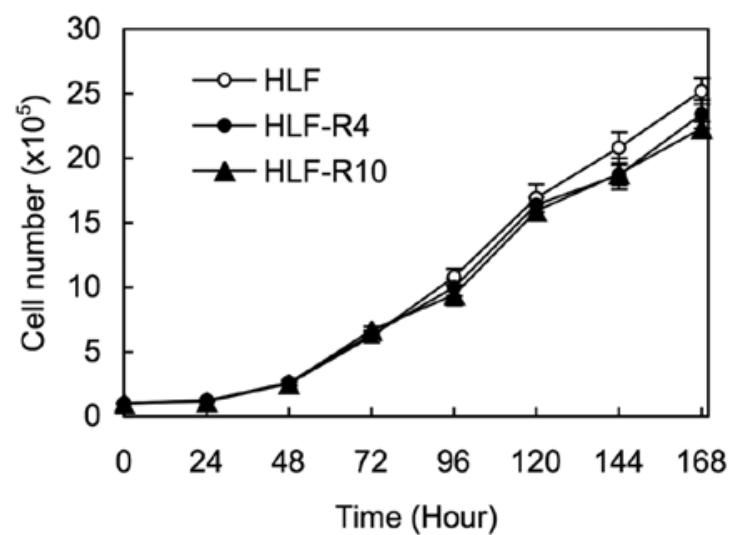

Figure 1. Cell growth assay. The experiments were carried out for $168 \mathrm{~h}$, with counting of the number of cells every $24 \mathrm{~h}$. The proliferation rates of the HLF-R4 (•) and HLF-R10 (৯) cells are similar to that of the HLF cells (O). The results are expressed as the means \pm standard error of the mean values from three assays.

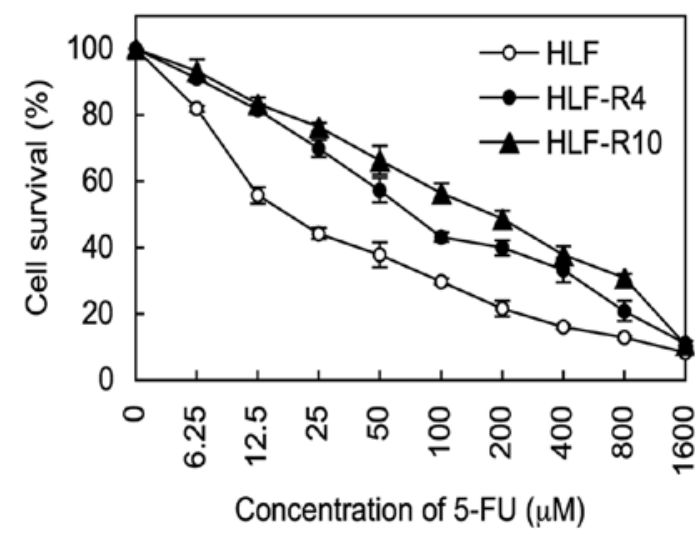

Figure 2. Chemosensitivity assay. $\mathrm{IC}_{50}$ values of 5-FU for the HLF (O), HLF-R4 (•), and HLF-R10 (^) cells are 17.92 $\mu \mathrm{M}, 69.80 \mu \mathrm{M}$ and $193.47 \mu \mathrm{M}$, respectively. Therefore, the 5-FU resistance of the HLF-R4 cells is 3.9-fold, and that of the HLF-R10 cells is 10.8-fold greater than that of the HLF cells. The results are expressed as the means \pm standard error of the mean values from three assays.

found that mRNA expression of ribonucleotide reductases $(R N R-R 1, R N R-R 2)$ was down-regulated and multi-drug resistance protein 5 (MRP5) was up-regulated in the HLF-Rs

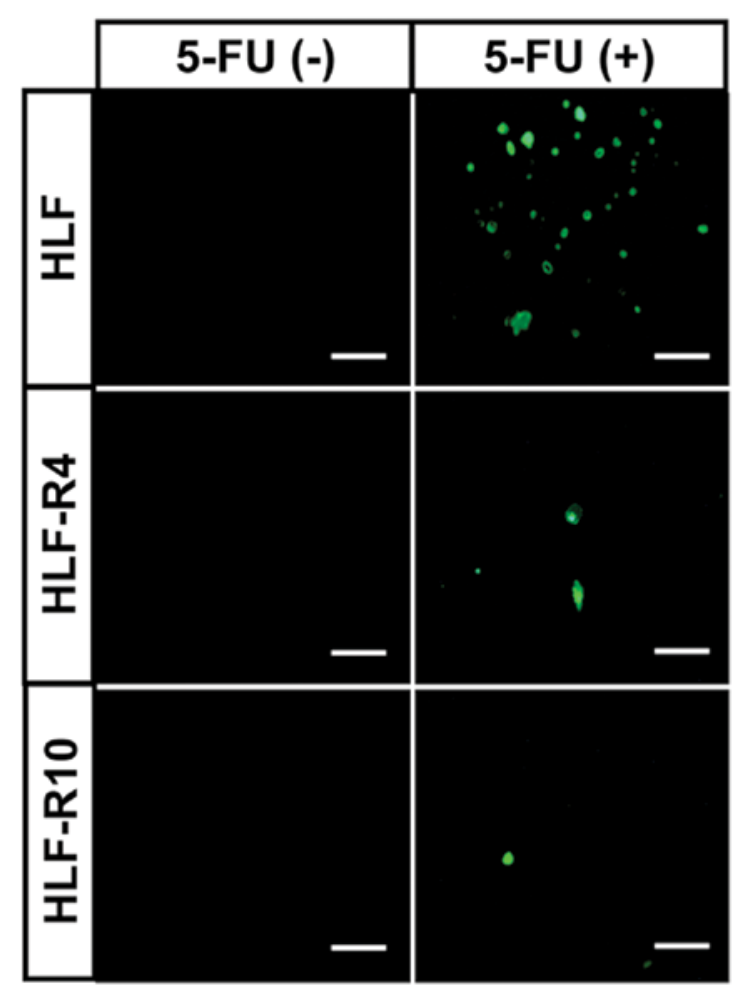

Figure 3. TUNEL assay. To evaluate the effect of 5-FU on the HLF, HLF-R4, and HLF-R10 cells, a TUNEL assay was used to confirm the apoptotic changes. TUNEL staining shows a dramatic increase in the number of cells that were stained green, which indicates that apoptosis occurred in HLF cells exposed to 5-FU. 5-FU-induced apoptosis is inhibited in the HLF-R4 and HLF-R10 cells. The experiments were repeated three times with similar results. Scale bar = $50 \mu \mathrm{m}$.

cells in a 5-FU-chemoresistant level-dependent manner (Fig. 5) $(\mathrm{p}<0.05)$.

\section{Discussion}

5-FU is key anticancer chemotherapy used to treat solid tumors, such as gastric, colorectal, pancreas, breast, and lung carcinomas (21-25). 5-FU also has been preferentially used alone or combined with other chemotherapeutic drugs for HCCs $(2,3)$. Although several mechanisms of 5-FU resistance have been investigated, no one mechanism completely explains the clinical response to 5-FU chemotherapy. Since it is extremely important to understand the resistance mechanism in order to develop better treatments, establishing 5-FU-resistant cells was 

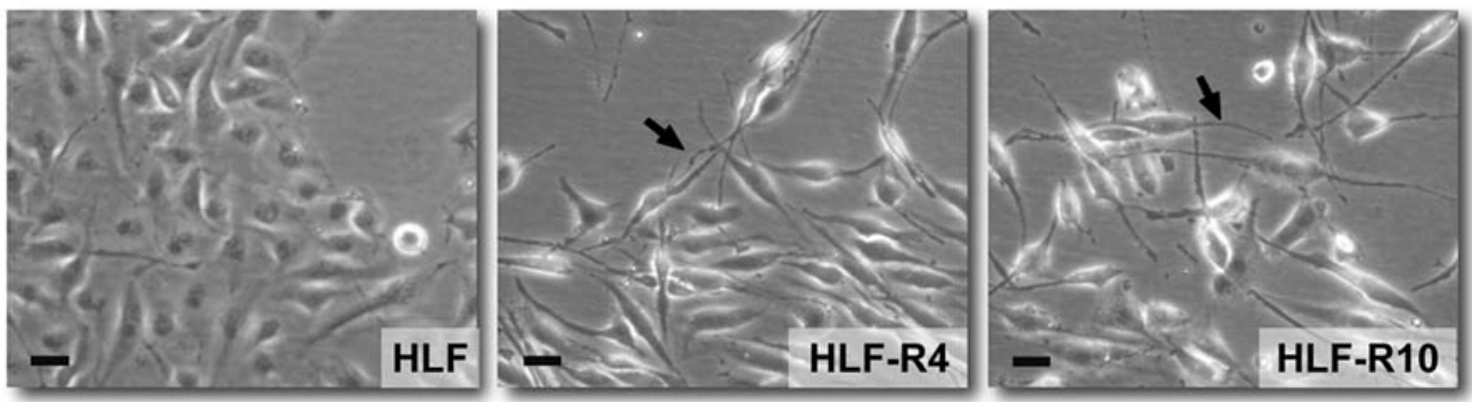

Figure 4. Cell morphology. 5-FU-resistant cells (HLF-R4 and HLF-R10) are morphologically distinct from their parental cell line, HLF. The resistant cells have loss of cell-cell adhesion, spindle-shaped morphology, and increased formation of pseudopodia (arrow). Scale bar, $20 \mu \mathrm{m}$.
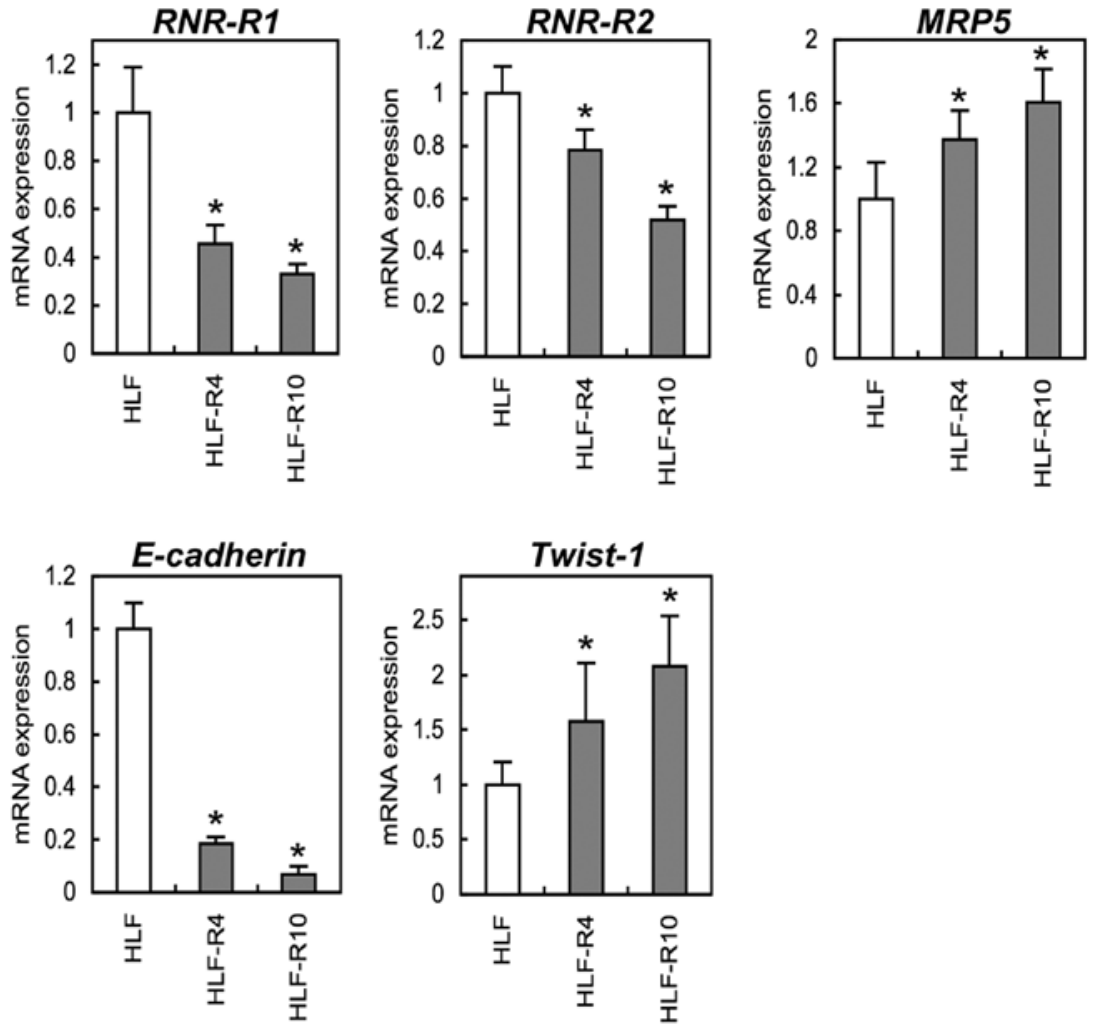

Figure 5. mRNA expression analysis. qRT-PCR was performed to investigate mRNA levels of RNR-R1, RNR-R2, MRP5, E-cadherin, and Twist-1. PCR shows up-regulation of MRP5 and Twist-1 and down-regulation of $R N R-R 1, R N R-R 2$, and E-cadherin dependent on the level of 5-FU chemoresistance. Data are expressed as the means \pm standard error of the mean values from three assays ( $\mathrm{p}<0.05$ by the Mann-Whitney $\mathrm{U}$ test).

indispensable to this type of study. Cell lines that are resistant to 5-FU have been established from several cancers $(6,26-28)$. However, to date, only the Bel7402/5-FU cell line from HCC has been reported to be resistant to 5-FU (29). To obtain further detailed information on 5-FU resistance in $\mathrm{HCC}$, in the current study we evaluated two new 5-FU-resistant cell lines, HLF-R4 and HLF-R10, from an undifferentiated HCC cell line that showed different resistance levels to 5-FU.

Consistent with previous reports that showed that 5-FU metabolism and activity of 5-FU transport were closely related to 5-FU resistance $(30,31)$, the current data showed downregulated $R N R s$ and up-regulated MRP5 genes. RNR, a key enzyme in 5-FU metabolism, catalyzed 5-fluorouridine diphosphate to 5-fluorodeoxyuridine diphosphate, the main precursor of 5-fluorodeoxyuridine monophosphate (FdUMP) (32).
Decreased RNR activity leads to a low FdUMP level followed by inhibited thymidylate synthase cellular activity, indicating that acquired resistance to 5-FU occurs in the cells (6-10). MRP5, an energy-dependent ATP-binding cassette transporter protein (33), mediates the ATP-dependent transport of various substrates, such as monophosphate metabolites (30), across biologic membranes (34) and is responsible for broad-spectrum resistance to chemotherapy including 5-FU. 5-FU metabolism may contribute to a basic understanding of the molecular mechanisms of chemoresistance. Further study is necessary to identify other mechanisms.

In addition to typical morphologic phenotypes of EMT in the HLF-Rs cells, we found decreased E-cadherin expression caused by Twist-1 expression, which is consistent with the results of Matsuo et al (35). These findings reflected an important process 
by which cancer cells may potentially become chemoresistant. Even though the relationship between EMT and chemoresistance remains unclear, induction of EMT in 5-FU-chemoresistant HCC cells might represent a new potentially exciting research area into 5-FU-resistance mechanisms. Thus, EMT is likely to be a potential therapeutic target for development of anticancer drugs in HCCs. Our data should eventually benefit patients with advanced HCC or 5-FU-chemoresistant HCCs for whom there currently are few effective treatment options.

In conclusion, we found that alterations of enzymes affecting 5-FU transport and metabolism as well as EMT were observed in the HLF-Rs. Nevertheless, it seems likely that multiple mechanisms may lead to 5-FU resistance. To the best of our knowledge, the Bel7402/5-FU cell line showed no EMT changes (29), 5-FU-resistant cell line associated with EMT is clearly required in HCC. Therefore, the HLF-Rs cells might be useful in vitro models for understanding 5-FU-resistant mechanisms in HCCs. Further, the HLF-R4 and HLF-R10 cells have different degrees of 5-FU resistance, so there are advantages to investigating the process of 5-FU resistance.

\section{Acknowledgements}

We thank Dr Mamoru Nukatsuka (Taiho Pharmaceutical Co., Ltd.) for helpful discussion and Yuhki Suzuki, Chisato Kitayama, and Rina Ito (Department of Drug Information and Communication, Graduate School of Pharmaceutical Sciences, Chiba University) for their considerable contributions to cell culture in the process of establishment of the HLF-Rs. We also thank Lynda C. Charters for editing this manuscript.

\section{References}

1. Kamangar F, Dores GM and Anderson WF: Patterns of cancer incidence, mortality, and prevalence across five continents: defining priorities to reduce cancer disparities in different geographic regions of the world. J Clin Oncol 24: 2137-2150, 2006.

2. Wang XN, Han X, Xu LN, Yin LH, Xu YW, Qi Y and Peng JY: Enhancement of apoptosis of human hepatocellular carcinoma SMMC-7721 cells through synergy of berberine and evodiamine. Phytomedicine 15: 1062-1068, 2008.

3. Ming ZJ, Hu Y, Qiu YH, Cao L and Zhang XG: Synergistic effects of beta-aescin and 5-fluorouracil in human hepatocellular carcinoma SMMC-7721 cells. Phytomedicine 17: 575-580, 2010.

4. Higuchi E, Oridate N, Furuta Y, Suzuki S, Hatakeyama H, Sawa H, Sunayashiki-Kusuzaki K, Yamazaki K, Inuyama Y and Fukuda S: Differentially expressed genes associated with CIS-diamminedichloroplatinum (II) resistance in head and neck cancer using differential display and CDNA microarray. Head Neck 25: 187-193, 2003.

5. Scotto KW and Bertino JR: Natural and acquired resistance to chemotherapeutic agents. In: The Molecular Basis of Cancer, 2nd edition. W. B. Sanders, Foster City, pp407-422, 2001.

6. Johnston PG, Drake JC, Trepel J and Allegra CJ: Immunological quantitation of thymidylate synthase using the monoclonal antibody TS 106 in 5-fluorouracil-sensitive and -resistant human cancer cell lines. Cancer Res 52: 4306-4312, 1992.

7. Aschele C, Sobrero A, Faderan MA and Bertino JR: Novel mechanism(s) of resistance to 5-fluorouracil in human colon cancer (HCT-8) sublines following exposure to two different clinically relevant dose schedules. Cancer Res 52: 1855-1864, 1992.

8. Copur S, Aiba K, Drake JC, Allegra CJ and Chu E: Thymidylate synthase gene amplification in human colon cancer cell lines resistant to 5-fluorouracil. Biochem Pharmacol 49: 1419-1426, 1995.

9. Berger SH, Jenh CH, Johnson LF and Berger FG: Thymidylate synthase overproduction and gene amplification in fluorodeoxyuridine-resistant human cells. Mol Pharmacol 28: 461-467, 1985.
10. Berger SH and Berger FG: Thymidylate synthase as a determinant of 5-fluoro-2'-deoxyuridine response in human colonic tumor cell lines. Mol Pharmacol 34: 474-479, 1988.

11. Jin S and Scotto KW: Transcriptional regulation of the MDR1 gene by histone acetyltransferase and deacetylase is mediated by NF-Y. Mol Cell Biol 18: 4377-4384, 1998.

12. Yang AD, Fan F, Camp ER, van Buren G, Liu W, Somcio R, Gray MJ, Cheng H, Hoff PM and Ellis LM: Chronic oxaliplatin resistance induces epithelial-to-mesenchymal transition in colorectal cancer cell lines. Clin Cancer Res 12: 4147-4153, 2006.

13. Shah AN, Summy JM, Zhang J, Park SI, Parikh NU and Gallick GE: Development and characterization of gemcitabine-resistant pancreatic tumor cells. Ann Surg Oncol 14: 3629-3637, 2007.

14. Thiery JP: Epithelial-mesenchymal transitions in tumour progression. Nat Rev Cancer 2: 442-454, 2002.

15. Thiery JP and Chopin D: Epithelial cell plasticity in development and tumor progression. Cancer Metastasis Rev 18: 31-42, 1999.

16. Elliott BE, Hung WL, Boag AH and Tuck AB: The role of hepatocyte growth factor (scatter factor) in epithelial-mesenchymal transition and breast cancer. Can J Physiol Pharmacol 80: 91-102, 2002.

17. Chung YM, Park S, Park JK, Kim Y, Kang Y and Yoo YD: Establishment and characterization of 5-fluorouracil-resistant gastric cancer cells. Cancer Lett 159: 95-101, 2000.

18. Negoro K, Yamano Y, Fushimi K, Saito K, Nakatani K, Shiiba M, Yokoe H, Bukawa H, Uzawa K, Wada T, Tanzawa H and Fujita S: Establishment and characterization of a cisplatin-resistant cell line, KB-R, derived from oral carcinoma cell line, KB. Int J Oncol 30: 1325-1332, 2007.

19. Nakamura M, Nakatani K, Uzawa K, Ono K, Uesugi H, Ogawara K, Shiiba M, Bukawa H, Yokoe H, Wada T, Fujita S and Tanzawa H: Establishment and characterization of a cisplatin-resistant oral squamous cell carcinoma cell line, H-1R. Oncol Rep 14: 1281-1286, 2005.

20. Nakatani K, Nakamura M, Uzawa K, Wada T, Seki N, Tanzawa H and Fujita S: Establishment and gene analysis of a cisplatin-resistant cell line, Sa-3R, derived from oral squamous cell carcinoma. Oncol Rep 13: 709-714, 2005.

21. Lee JH, Kim MC, Oh SY, Kwon HC, Kim SH, Kwon KA, Lee S, Jeong JS, Choi SR and Kim HJ: Predictive value of in vitro adenosine triphosphate-based chemotherapy response assay in advanced gastric cancer patients who received oral 5-Fluorouracil after curative resection. Cancer Res Treat 43: 117-123, 2011.

22. Gamelin EC, Danquechin-Dorval EM, Dumesnil YF, Maillart PJ, Goudier MJ, Burtin PC, Delva RG, Lortholary AH, Gesta PH and Larra FG: Relationship between 5-fluorouracil (5-FU) dose intensity and therapeutic response in patients with advanced colorectal cancer receiving infusional therapy containing 5-FU. Cancer 77: 441-451, 1996.

23. Murakami Y, Uemura K, Sudo T, Hayashidani Y, Hashimoto Y, Ohge $\mathrm{H}$ and Sueda T: Impact of adjuvant gemcitabine plus S-1 chemotherapy after surgical resection for adenocarcinoma of the body or tail of the pancreas. J Gastrointest Surg 13: 85-92, 2009.

24. Uday CK, Devarayalu BVSK and Mahmood S: Overall survival rate in breast cancer patients treated with 5-fluorouracil: a review of randomized clinical trials. Int $\mathbf{J}$ Pharmaceut Biomed Res 1: 132-135, 2010.

25. Huang CL, Yokomise H, Kobayashi S, Fukushima M, Hitomi S and Wada $\mathrm{H}$ : Intratumoral expression of thymidylate synthase and dihydropyrimidine dehydrogenase in non-small cell lung cancer patients treated with 5-FU-based chemotherapy. Int J Oncol 17: 47-54, 2000.

26. Plasencia C, Rooney PH, Taron M, Martinez-Balibrea E, McLeod HL and Abad A: Chromosomal imbalance maps of human 5FU-resistant colorectal cancer cell lines: implications in the analysis of 5FU-acquired resistance mechanisms. Int J Oncol 22: 945-953, 2003.

27. Tanaka T, Bai T and Toujima S: Establishment and characterization of monoclonal 5-fluorouracil-resistant cell lines derived from human endometrial adenocarcinoma. Int J Oncol 37: 731-736, 2010.

28. Wen J, Zheng B, Hu Y, Zhang X, Yang H, Luo KJ, Li YF and Fu JH: Establishment and biological analysis of the EC109/CDDP multidrug-resistant esophageal squamous cell carcinoma cell line. Oncol Rep 22: 65-71, 2009.

29. Huang M and Liu G: The study of innate drug resistance of human hepatocellular carcinoma Bel7402 cell line. Cancer Lett 135: 97-105, 1999. 
30. Pratt S, Shepard RL, Kandasamy RA, Johnston PA, Perry W III and Dantzig AH: The multidrug resistance protein 5 (ABCC5) confers resistance to 5-fluorouracil and transports its monophosphorylated metabolites. Mol Cancer Ther 4: 855-863, 2005.

31. Nakano Y, Tanno S, Koizumi K, Nishikawa T, Nakamura K, Minoguchi M, Izawa T, Mizukami Y, Okumura T and Kohgo Y: Gemcitabine chemoresistance and molecular markers associated with gemcitabine transport and metabolism in human pancreatic cancer cells. Br J Cancer 96: 457-463, 2007.

32. Fukushima M, Fujioka A, Uchida J, Nakagawa F and Takechi T: Thymidylate synthase (TS) and ribonucleotide reductase (RNR) may be involved in acquired resistance to 5-fluorouracil (5-FU) in human cancer xenografts in vivo. Eur J Cancer 37: 1681-1687, 2001.
33. Hinoshita E, Uchiumi T, Taguchi K, Kinukawa N, Tsuneyoshi M, Maehara Y, Sugimachi K and Kuwano M: Increased expression of an ATP-binding cassette superfamily transporter, multidrug resistance protein 2 , in human colorectal carcinomas. Clin Cancer Res 6: 2401-2407, 2000.

34. Kruh GD and Belinsky MG: The MRP family of drug efflux pumps. Oncogene 22: 7537-7552, 2003.

35. Matsuo N, Shiraha H, Fujikawa T, Takaoka N, Ueda N, Tanaka S, Nishina S, Nakanishi Y, Uemura M, Takaki A, Nakamura S, Kobayashi Y, Nouso K, Yagi T and Yamamoto K: Twist expression promotes migration and invasion in hepatocellular carcinoma. BMC Cancer 9: 240-251, 2009. 